\title{
SPARSIFYING PRECONDITIONER FOR SOLITON CALCULATIONS
}

\author{
JIANFENG LU AND LEXING YING
}

\begin{abstract}
We develop a robust and efficient method for soliton calculations for nonlinear Schrödinger equations. The method is based on the recently developed sparsifying preconditioner combined with Newton's iterative method. The performance of the method is demonstrated by numerical examples of gap solitons in the context of nonlinear optics.
\end{abstract}

\section{INTRODUCTION}

1.1. Background. In this work, we consider a type of nonlinear equations associated with nonlinear eigenvalue problems: Given $\lambda$, we solve for $u$ such that

$$
-\Delta u+V u+N(x, u)=\lambda u
$$

with periodic boundary condition on a box domain $\mathbb{T}^{d}:=[0,1]^{d}$, where $d$ is the dimension. Here $V$ is some background potential and $N$ is the nonlinear term. This is a natural generalization of linear eigenvalue problem, for instance

$$
-\Delta u+V u=\lambda u
$$

with periodic boundary condition. Note that while for the linear eigenvalue problem (2), a nontrivial solution $u$ exists only when $\lambda$ is an eigenvalue and the normalization of $u$ is not fixed (the eigenvalue remains the same and the eigenfunction is simply scaled), the solution to the nonlinear problem (1) will have a fixed normalization due to the presence of the nonlinear term. In fact, for most cases, we are interested in the relation between $\lambda$ (which can be seen as a Lagrange multiplier) and the normalization $\|u\|_{2}$.

This type of nonlinear eigenvalue problems arises in several applications, for example in nonlinear photonic crystals (see e.g., $2,3,12$ 13 and the numerical examples in Section 3) and in the study of solitons in nonlinear wave equations, for instance the Gross-Pitaevskii equation. It is also related to electronic structure theory, for which a system of nonlinear eigenvalue problem arises as the Kohn-Sham equations. While we will focus on applications in nonlinear Schrödinger equations for solitons in photonic crystals in this work, the algorithm applies to other context as well.

Date: March 21, 2016.

The work of J.L. is supported in part by the Alfred P. Sloan Foundation and the National Science Foundation under grant DMS-1312659 and DMS-1454939. The work of L.Y. is partially supported by the National Science Foundation under grant DMS-1328230 and DMS-1521830 and the U.S. Department of Energy's Advanced Scientific Computing Research program under grant DE-FC02-13ER26134/DE-SC0009409. J.L. would like to thank helpful discussions with Jeremy Marzuola and Mikael Rechtsman. 
While this paper considers numerical methods to solve the equation 11 , let us mention that the analytical understanding of such equations is also a very active research area in applied mathematics, see e.g., a recent review [15].

1.2. Previous approaches. To get the nonlinear eigenvalue $\lambda$ and the corresponding state $u$, one popular approach in the engineering literature is to use an indirect strategy based on time evolution. To get an estimate of the eigenvalue, the time-dependent equation

$$
i u_{t}=\Delta u-V(u)-N(x, u)
$$

is evolved for a given time period with a random initial data; a Fourier transform in time is then used to extract the frequencies with significant amplification at the final time compared to the initial data. While the time evolution method is very useful to extract resonant frequencies for linear problems, this procedure becomes quite tricky if the nonlinear effects become significant (see e.g., [14]). In particular, the lack of the superposition principle makes it difficult to interpret the amplification in the Fourier space.

Thus, in the presence of nonlinearity, it is preferred to solve the nonlinear eigenvalue problem directly (without resorting to time-evolution) based on iterative methods. One such approach is the self-consistent iteration scheme (see [6 Chapter 9] for an introduction) based on formulating the problem as a linear eigenvalue problem at each iteration, e.g.,

$$
-\Delta u^{(n+1)}+V u^{(n+1)}+\frac{N\left(x, u^{(n)}\right)}{u^{(n)}} u^{(n+1)}=\lambda^{(n+1)} u^{(n+1)}
$$

by choosing $\lambda^{(n+1)}$ the closest eigenvalue to the desired $\lambda$. The difficulty of this type of method is that we do not have a priori knowledge on the normalization of $u^{(n+1)}$ (recall that the normalization is arbitrary at the linear level, but has to be fixed for the nonlinear equation), so that some heuristics or parameter tuning is needed.

Another more popular iterative scheme was proposed originally by Petviashvili [9] for equation of the type

$$
-\Delta u-u^{3}=\lambda u
$$

for $\lambda>0$, so that the $N(x, u)$ is the focusing cubic nonlinearity $-u^{3}$ and there is no potential $V$. The iteration scheme reads

$$
\widehat{u^{(n+1)}}(k)=M_{n}^{\gamma} \frac{\mathscr{F}\left(\left(u^{(n)}\right)^{3}\right)(k)}{\lambda+4 \pi^{2}|k|^{2}},
$$

where $M_{n}$ is a stabilizing factor given by

$$
M_{n}=\frac{\sum_{k}\left(\lambda+4 \pi^{2}|k|^{2}\right)\left|\widehat{u^{(n)}(k)}\right|^{2}}{\sum_{k} \widehat{u^{(n)}(k)} \mathscr{F}\left(\left(u^{(n)}\right)^{3}\right)(k)},
$$

and $\gamma$ is a parameter to be chosen so that the iteration converges. Here $\widehat{u}$ and $\mathscr{F}[u]$ both denote the Fourier transform of $u$ : For each $k \in \mathbb{Z}^{d}$,

$$
\widehat{u}(k)=\mathscr{F}[u](k):=\int_{\mathbb{U}^{d}} e^{-2 \pi i(k \cdot x)} u(x) \mathrm{d} x .
$$


The convergence of the Pitviashvili scheme has been analyzed in [8]. The iteration scheme was also generalized to deal with more general nonlinearities and the case with potential terms, see for example [1 5] and references therein. All methods of this type reformulate the nonlinear problem (1) using the Fourier space representation, in which the positively shifted Laplacian operator becomes diagonal, and hence can be explicitly inverted.

1.3. Our contribution. In this work, to solve the nonlinear equation [1], we will use the standard Newton iteration method. Given $\lambda$, to find the eigenfunction $u$ (and hence its normalization), the Newton iterative scheme is given by

$$
u^{(n+1)}=u^{(n)}-\left(-\Delta+L_{u^{(n)}}-\lambda\right)^{-1}\left(-\Delta u^{(n)}+V u^{(n)}+N\left(x, u^{(n)}\right)-\lambda u^{(n)}\right) .
$$

Here $L_{u}$ is the linearization of the potential terms at $u: L_{u}=V+\frac{\delta N}{\delta u}$. The key step for the above iteration scheme is the solution to the linear system

$$
\left(-\Delta+L_{u}-\lambda\right) v=r
$$

for the current iterate $u$ and remainder denoted as $r$.

We will consider the Fourier pseudospectral method to discretize [1, which is very popular for the nonlinear wave equations with periodic boundary conditions. The pseudospectral method typically requires minimal degree of freedoms for a given accuracy among standard discretizations and is also easy to implement, and hence widely used in physics and engineering literature (see e.g., [10 in the context of photonics). As we have discussed already, most previous iterative methods are built on the Fourier pseudospectral method.

The drawback of the pseudo-spectral method however is that the discrete matrices resulting from discretizing $-\Delta+L_{u}-\lambda$ are dense, so that it is usually very expensive to directly solve equations like [10). Therefore, some iterative methods have to be used, in which a shifted Laplacian is often used as a preconditioner. In fact, the iterative methods introduced by Petviashvili [9] and the various generalizations can be understood as using the shifted Laplacian in a way that the outer and inner iterations are combined.

The main motivation of this work is that, rather than finding an alternative iterative scheme which usually is less effective than the Newton iteration, we will instead use an efficient way to solve the dense discrete system resulting from (10). The key component is the recently developed sparsifying preconditioner [16], which transforms the discrete dense systems numerically to a sparse one. Sparse direct solvers can then be combined with standard iterative schemes to provide an efficient way to solve the linear system [10, and hence the nonlinear equation.

\section{AlgorithM DESCRIPTION}

2.1. Sparsifying preconditioner. Let us focus on numerical solution of 10 , given the current iterate $u$ and hence $L_{u}$. We assume the nonlinearity term $N(x, u)$ only depends locally on $u$, and hence the derivative $\frac{\delta N}{\delta u}$ with respect to $u$ gives a potential-type term in (10). 
We assume that the computation domain is the periodic unit box $[0,1]^{d}$ and discretize the problem using the Fourier pseudospectral method. Let us index the set of all grid points as

$$
J=\left\{\left(j_{1}, \ldots, j_{d}\right) \mid 0 \leq j_{1}, \ldots, j_{d}<n\right\} .
$$

For each $j \in J$, in the pseudospectral method, the grid point is given by

$$
x_{j}=j h
$$

with $h=\frac{1}{n}$ being the mesh size in each dimension. The function $v$ (and similarly $u$ and $r$, etc.) is hence discretized as vectors with

$$
v_{j}=v(j h)
$$

The corresponding Fourier grid is then given by

$$
K=\left\{\left(k_{1}, \ldots, k_{d}\right) \mid-n / 2 \leq k_{1}, \ldots, k_{d}<n / 2\right\} .
$$

In the pseudospectral method, the Laplacian operator is discretized as (with slight abuse of notation, we still denote by $-\Delta$ the discretized operator)

$$
-\Delta=F^{-1} \operatorname{diag}\left(4 \pi^{2}|k|^{2}\right)_{k \in K} F,
$$

where $F$ and $F^{-1}$ are the discrete Fourier and inverse Fourier transforms

$$
\begin{aligned}
(F f)_{k} & =\frac{1}{n^{d}} \sum_{j \in J} e^{-2 \pi i(k \cdot j) / n} f_{j}, \quad k \in K ; \\
\left(F^{-1} g\right)_{j} & =\sum_{k \in K} e^{2 \pi i(j \cdot k) / n} g_{k}, \quad j \in J .
\end{aligned}
$$

So that $-\Delta$ is diagonal in the Fourier space. The discretized $L_{u}$ (again, we use the same notation after discretization), on the other hand, is a diagonal matrix in the physical space,

$$
L_{u}=\operatorname{diag}\left(L_{u}(j h)\right)_{j \in J} .
$$

Following the notations introduced in in [13, (15), and [18, the discretized problem of [10) takes the same form

$$
\left(-\Delta+L_{u}-\lambda\right) v=r
$$

We will briefly recall the key idea of the sparsifying preconditioner for solving this type of equations. More details can be found in [16] (see also [17]).

Denote $l=\left\langle L_{u}\right\rangle$ the spatial average of $L_{u}$. We assume without loss of generality that $-\Delta+l-\lambda$ is invertible on $\mathbb{T}^{d}$ with periodic boundary condition, otherwise, we will use a slight perturbation of $\lambda$ instead and put the difference into $L_{u}$. This allows us to rewrite 10 trivially as

$$
\left(-\Delta+(l-\lambda)+\left(L_{u}-l\right)\right) v=r
$$

The inverse $G$ of the constant part $(-\Delta+(l-\lambda))$ is given by

$$
G=F^{-1} \operatorname{diag}\left(\frac{1}{4 \pi^{2}|k|^{2}+l-\lambda}\right)_{k \in K} F
$$


Applying $G$ to both sides of 10 gives an equivalent linear system

$$
\left(I+G\left(L_{u}-l\right)\right) v=G r .
$$

Note that in the pseudo-spectral discretization, since $G$ can be easily evaluated by fast Fourier transform and $L_{u}-l$ is a diagonal matrix, applying the operators on both sides of 21) can be carried out efficiently. The main idea of the sparsifying preconditioner is to introduce a special sparse matrix $Q$ and multiply $Q$ on the both hand sides of 21]:

$$
\left(Q+Q G\left(L_{u}-l\right)\right) v=Q(G r) .
$$

The operator $Q$ is required to satisfy two requirements.

- First, the matrix $Q$ should correspond to a local stencil. More precisely, for each point $j$ the row $Q(j,:)$ is supported in a local neighborhood $\mu(j)$ (to be defined below);

- Second, the product $Q G$ should be negligible outside the support of the matrix $Q$. This requirement would allow $Q G$ to be well approximated by a sparse matrix with the same support pattern as $Q$. More precisely, for each point $j$, it is required that $(Q G)\left(j, \mu(j)^{c}\right)=$ $Q(j, \mu(j)) G\left(\mu(j), \mu(j)^{c}\right) \approx 0$.

Let $S=\{(j, \mu(j)), j \in J\}$ be the support of the sparse matrix $Q$. The second condition ensures that the product $Q G$ is also essentially supported on $S$. The sum $Q+Q G\left(L_{u}-l\right)$ is essentially supported in $S$ as well, since $\left(L_{u}-l\right)$ is a diagonal matrix. Define $P$ to be the restriction of $Q+$ $Q G\left(L_{u}-l\right)$ to $S$ by thresholding other values to zero, i.e.

$$
P_{i j}= \begin{cases}\left(Q+Q G\left(L_{u}-l\right)\right)_{i j}, & (i, j) \in S, \\ 0, & (i, j) \notin S .\end{cases}
$$

Knowing that $P$ is a close approximation of $Q+Q G\left(L_{u}-l\right)$, one arrives at the approximate equation

$$
P v \approx Q(G r) .
$$

The sparsifying preconditioner computes an approximate solution $\tilde{v}$ by solving

$$
P \tilde{v}=Q(G r) .
$$

Since $P$ is sparse, the above equation can be solved by sparse direct solvers such as the nested dissection algorithm [4]. The nested dissection algorithm constructs an efficient sparse factorization of $P^{-1}$ by exploiting the sparse pattern of $P$. Once the factorization is ready, applying $P^{-1}$ to a given factor can be done efficiently. The solution $\tilde{v}=P^{-1} Q G r$ can be used as a preconditioner for the standard iterative algorithms such as GMRES [11] for the solution of [19].

As detailed in [16], the neighborhood $\mu(j)$ in the first condition is chosen to be the stencil support of a standard spectral element method so that one can fully exploit the efficiency of the nested dissection method used for computing the approximate inverse for 22. 


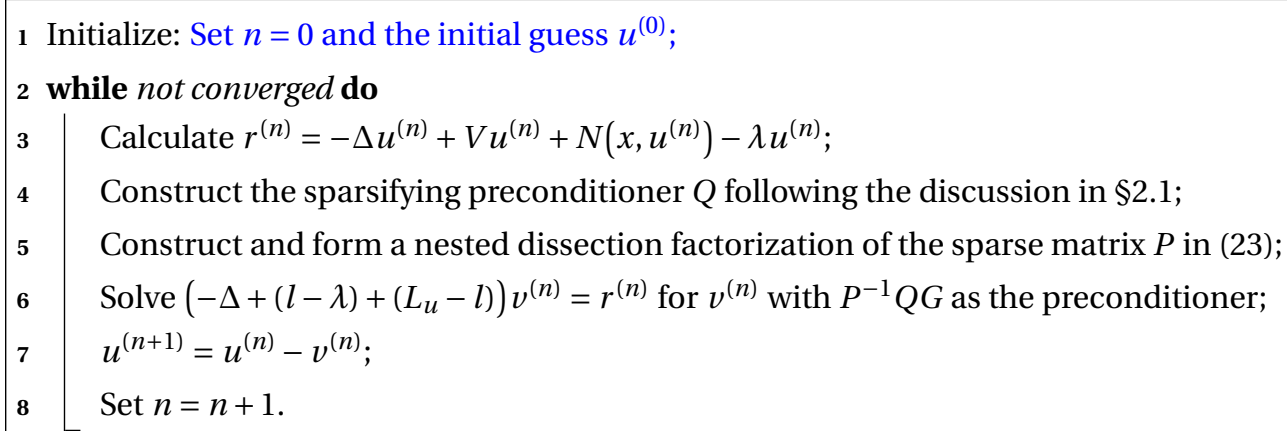

Algorithm 1: Newton iteration combined with sparsifying preconditioner.

2.2. Newton iteration. With the solution of (10) as discussed above, we solve the nonlinear equation (1) using the standard Newton iteration. We summarize here the overall algorithm as Algorithm 1

In practice, we choose the stopping criteria of the Newton iteration as

$$
\frac{\left\|u^{(n+1)}-u^{(n)}\right\|}{\left\|u^{(n)}\right\|} \leq 10^{-6}
$$

and we take a small tolerance $\left(10^{-10}\right)$ for the GMRES iteration for solving the linear equation in Step 6. As the overall method is based on the Newton's method, the convergence follows from the standard analysis (see for example [7. Chapter 3]), and we will not go into details here.

\section{NUMERICAL EXAMPLES}

To test the Algorithm 1] we consider a few examples from the optics, drawn from the paper [3]. Up to a rescaling of the domain and various parameters and variables, these nonlinear eigenvalue problem are of the type (1). The algorithms presented above are implemented in MATLAB and the results reported in this section are obtained on a Linux computer with a $2.6 \mathrm{GHz} \mathrm{CPU}$.

One example is Kerr nonlinearity, the equation is given by (using the notations of [3])

$$
-\frac{1}{2} \Delta u+\frac{V_{0}}{2}\left[\sin ^{2}(\pi x)+\sin ^{2}(\pi y)\right] u-\sigma|u|^{2} u=\lambda u,
$$

where $\sigma= \pm 1$ corresponds to focusing and defocusing cases. The other example we consider is the case of saturable nonlinearity:

$$
-\frac{1}{2} \Delta u+\frac{V_{0} u}{1+A^{2} \cos ^{2}(\pi x) \cos ^{2}(\pi y)+|u|^{2}}=\lambda u .
$$

In both examples, the computational domain is taken to be sufficiently large in order to make sure that the calculation is not suffered from finite size artifacts.

The particular interesting cases are when the eigenvalue (denoted by $\lambda$ in above equations) lies in the band gaps of the linear operator (the Bloch-Floquet theory applies thanks to the periodic structure). In the literature, the solutions are referred as gap solitons. From a mathematical point of view, this suggests that the operator on the left hand side of 10 is indefinite, which contains both positive and negative eigenvalues. Thus, the negatively shifted Laplacian will not be 
a good preconditioner in this case, which makes Pitviashvili type iterative methods very difficult to converge. In comparison, the Newton iteration captures the correct spectral behavior of the linearized operator, and is hence much more robust.

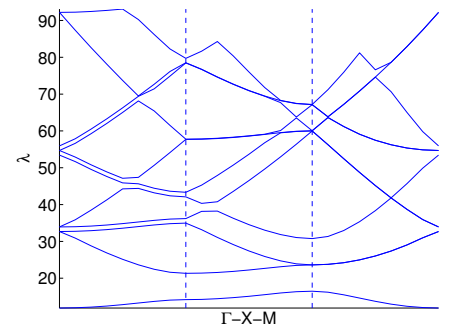

(a)

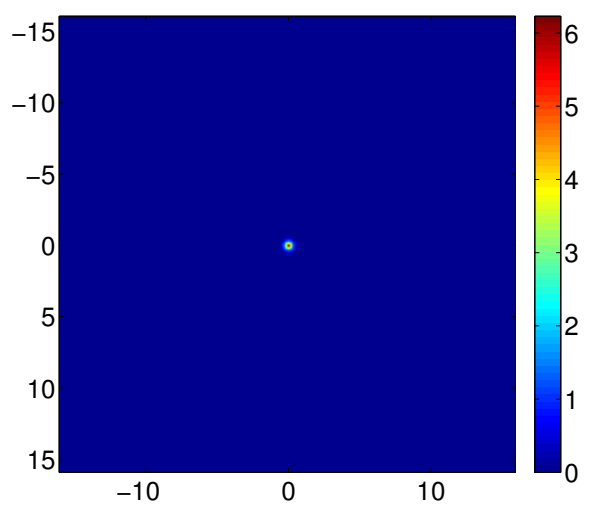

(d)

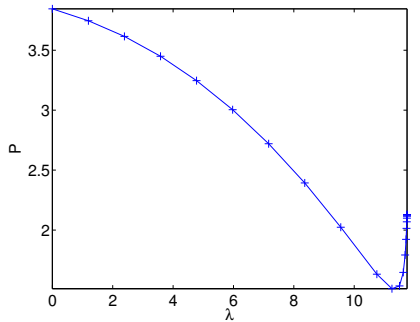

(b)

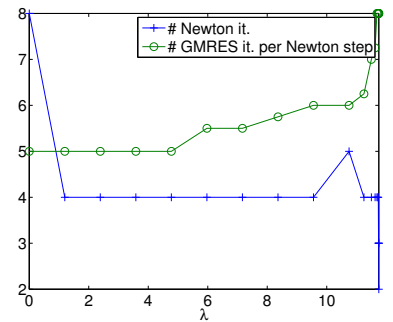

(c)

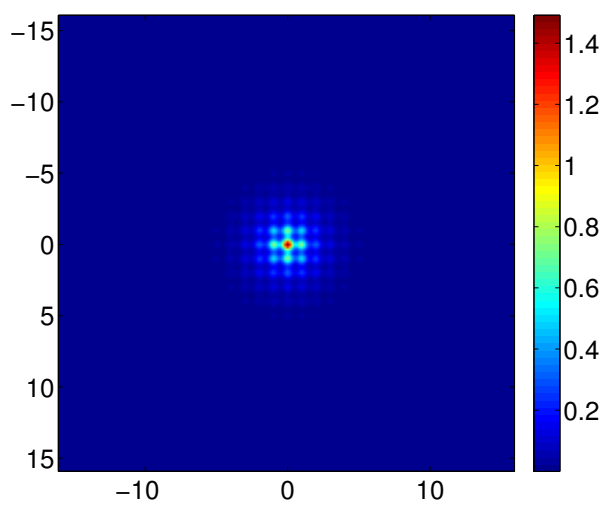

(e)

FIGURE 1. Kerr nonlinearity focusing case $V_{0}=28.8$. (a) the band structure of the linear problem. (b) the $\lambda-P$ curve. (c) the number of Newton iterations for each $\lambda$ value and the averaged number of preconditioned GMRES iteration for each Newton step. (d) the field profile at $\lambda=0$. (e) the field profile at $\lambda=$ 11.7498 .

Figure 1 summarizes the results of [26] in the focusing case $(\sigma=1)$ with $V_{0}=28.8$. The computation is carried out in the periodic domain $[-16,16]^{2}$ with 6 points per unit length. For the linear problem, the band structure is given in Figure 1(a). Figure 1 (b) plots the relationship between the eigenvalue $\lambda$ of the soliton and the power $P$ of the solution defined by $\int|u(x)|^{2} d x$. This $\lambda-P$ curve is obtained with a continuation path that starts from $\lambda=0$ and gradually grows to $\lambda \approx 11.75$. The range of $\lambda$ hence lies below the spectrum of the linearized operator in this example. At $\lambda=0$, the initial guess for $u(x)$ is chosen to be a localized Gaussian function with power $P=4$. For the following $q$ values, the solution of $u(x)$ of the previous $\lambda$ value is used as the initial guess of the Newton solve. Figure 1 (c) plots for each $\lambda$ value the number of Newton iterations as well as the averaged number of preconditioned GMRES iterations for each Newton step. Notice that, throughout the computation, the number of Newton iterations remains rather small and 
this demonstrates the effectiveness of the Newton's method and the continuation path. For each Newton step, the number of the preconditioned GMRES iterations stays quite small and this is a clear evidence of the effectiveness of the sparsifying preconditioner. For our implementation, the setup cost of the sparsifying preconditioner is about 4 seconds and each application of the sparsifying preconditioner takes about 0.02 seconds. Figure 1 (d) and (e) give the field profile $u(x)$ at $\lambda=0$ and $\lambda=11.7498$, respectively. The last plot shows that the support of the solution becomes quite extended as the eigenvalue $\lambda$ approaches the bottom boundary of the lowest energy band of the linear operator.

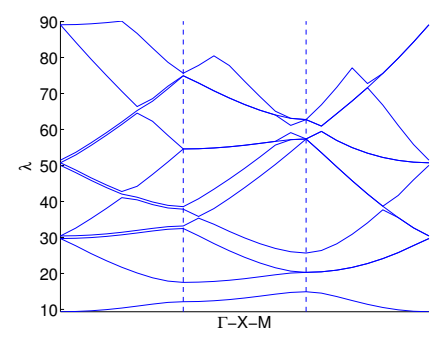

(a)

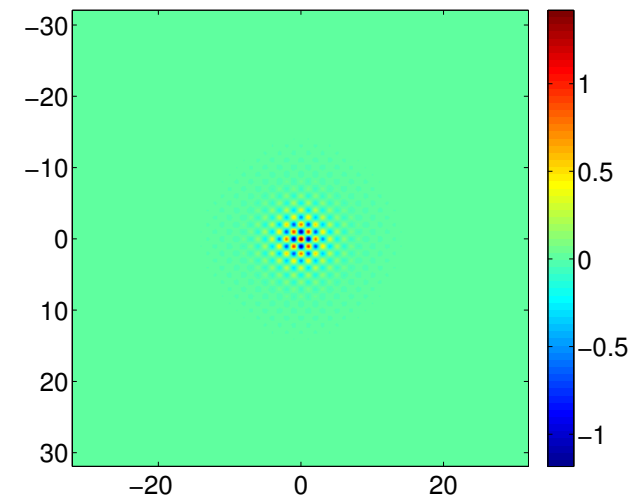

(d)

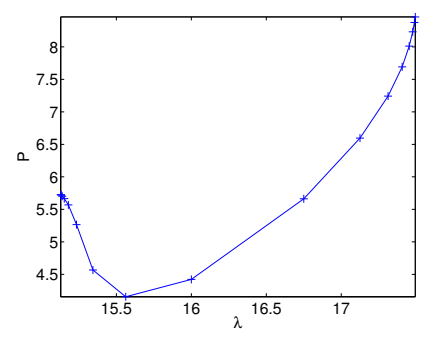

(b)

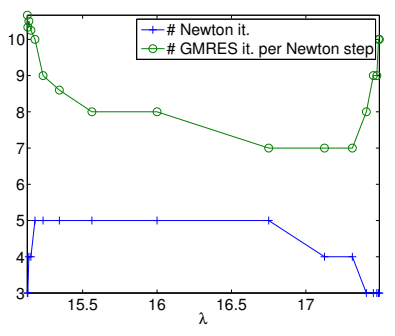

(c)

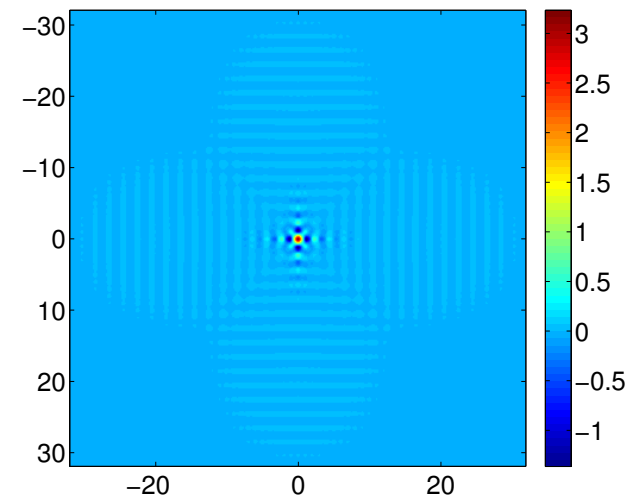

(e)

FIGURE 2 . Kerr nonlinearity defocusing case $V_{0}=21.6$. (a) the band structure of the linear problem. (b) the $\lambda-P$ curve. (c) the number of Newton iterations for each $\lambda$ value and the averaged number of preconditioned GMRES iteration for each Newton step. (d) the field profile at $\lambda=15.1284$. (e) the field profile at $\lambda=17.4941$.

Figure 2 shows the results of 26) in the defocusing case $(\sigma=-1)$ with $V_{0}=21.6$. Since the support of the defocusing gap soliton is significantly more extended, the computation domain $[-32,32]^{2}$ is used here with 6 points per unit length. The band structure of the linear problem is plotted in Figure 2(a). Figure 2(b) gives the $\lambda-P$ curve for $\lambda \in(15.125,17.5)$. This curve is obtained with two separated continuation paths, one from $\lambda=16$ down to $\lambda=15.125$ and the other from $\lambda=16$ to $\lambda=17.5$. As seen in the band structure, this range of $\lambda$ lies in between the 
first and second bands of the linearized operator. In both paths, the initial guess of $u(x)$ for $\lambda=16$ is a localized Gaussian function with power $P=4$. Figure 2(c) plots for each $\lambda$ the number of Newton iterations as well as the averaged number of preconditioned GMRES iterations for each Newton step. The curves show that our combination of the Newton's method, the continuation approach, and the sparsifying preconditioner results a efficient way to compute the gap solitons in the defocusing case. Due to the increase of the computational domain (as compared to the focusing case), the setup cost of the sparsifying preconditioner here is about 18 seconds and each application of the sparsifying preconditioner takes about 0.1 seconds. Figure 2 (d) and (e) show the field profile $u(x)$ at $\lambda=15.1284$ and $\lambda=17.4941$. In both plots, the essential support of $u(x)$ becomes extended since the eigenvalue $\lambda$ approaches the boundary of the nearby energy bands of the linear operator.

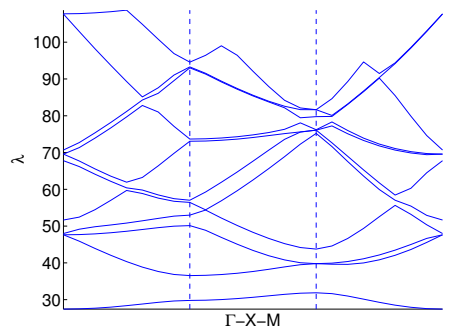

(a)

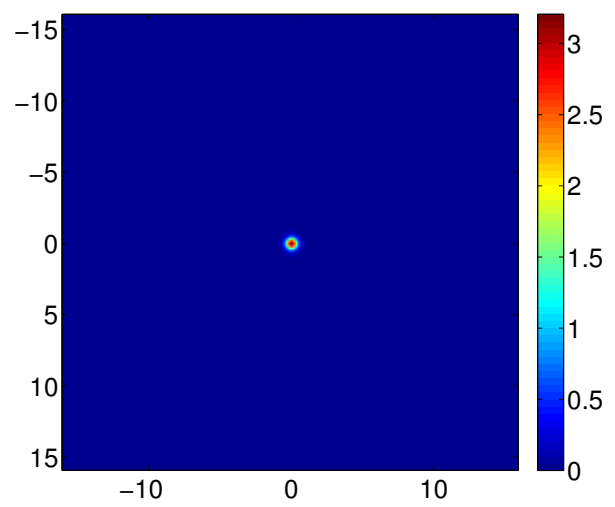

(d)

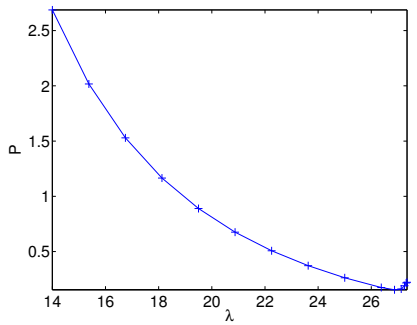

(b)

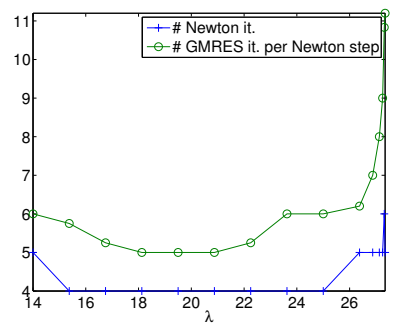

(c)

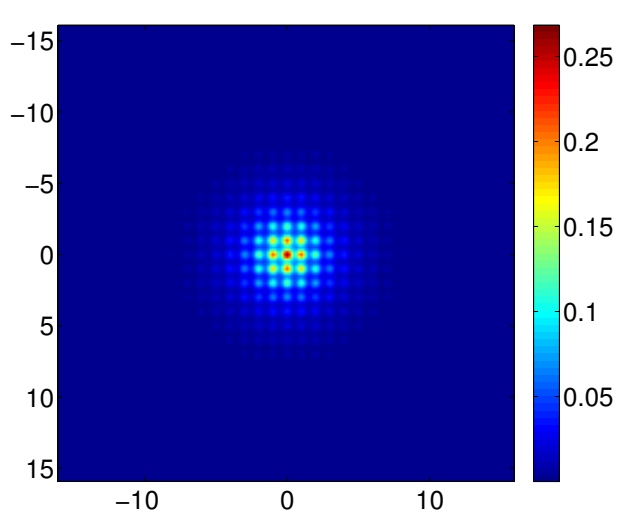

(e)

FIgURE 3. Saturable nonlinearity focusing case $V_{0}=36.3$ and $A=1.1$. (a) the band structure of the linear problem. (b) the $\lambda-P$ curve. (c) the number of Newton iterations for each $\lambda$ value and the averaged number of preconditioned GMRES iteration for each Newton step. (d) the field profile at $\lambda=14$. (e) the field profile at $\lambda=27.3438$.

Figure 3 summarizes the results of 27 in the focusing case with $V_{0}=36.3$ and $A=1$.1. For this case, the computational domain is set to be the periodic square $[-16,16]^{2}$ with 6 points per unit length. As before, Figure $3 \mathrm{a}$ (a) is the band structure of the linear problem. Figure $3(\mathrm{~b})$ is the 
$\lambda-P$ curve for $\lambda \in[14,27.375]$. The range of $\lambda$ lies below the spectrum of the linear problem. This curve is computed with a single continuation path that starts from $\lambda=14$. At $\lambda=14$, the initial guess for $u(x)$ is a localized Gaussian profile with power $P=2$. Figure $3(\mathrm{c})$ gives for each $\lambda$ value the number of Newton iterations and the averaged number of preconditioned GMRES iterations for each Newton step. Figure 3 (d) and (e) plot the field profile at $\lambda=14$ and $\lambda=27.3438$. Since the eigenvalue $\lambda=14$ is far from the energy band, the field profile $u(x)$ in Figure $3(\mathrm{c})$ is localized. On the contrary, $\lambda=27.3438$ approaches the lowest energy band of the linear operator from bottom, the field profile $u(x)$ is quite extended.

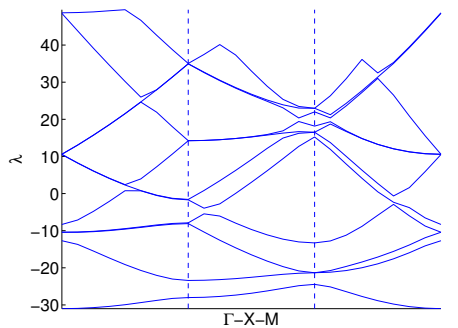

(a)

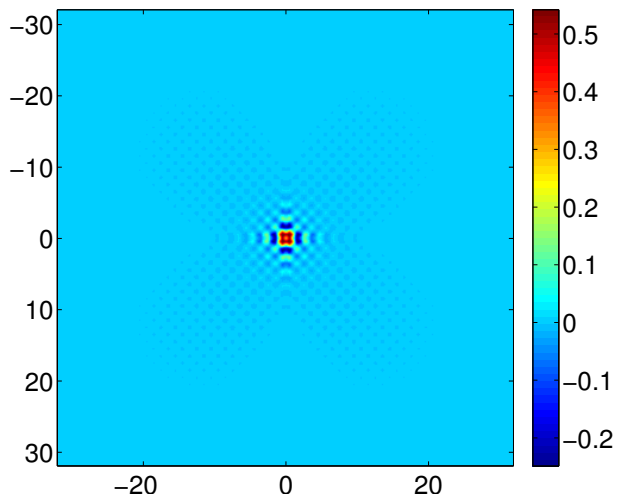

(d)

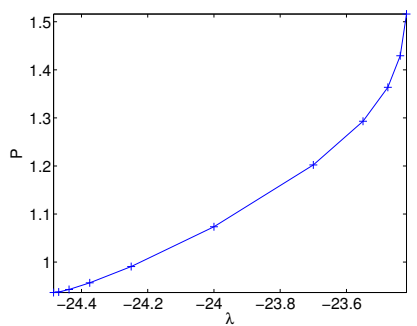

(b)

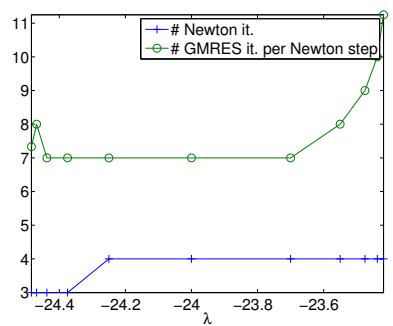

(c)

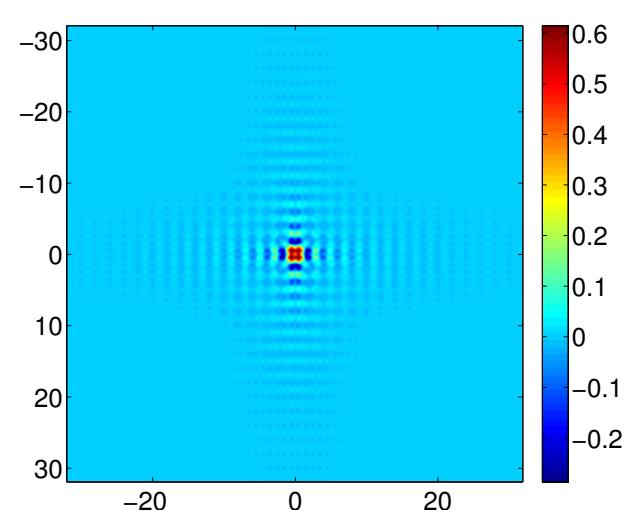

(e)

FiguRE 4. Saturable nonlinearity defocusing case $V_{0}=-36.3$ and $A=1.1$. (a) the band structure of the linear problem. (b) the $\lambda-P$ curve. (c) the number of Newton iterations for each $\lambda$ value and the averaged number of preconditioned GMRES iteration for each Newton step. (d) the field profile at $\lambda=-24.4844$. (e) the field profile at $\lambda=-23.4187$.

The results of (27) in the defocusing case with $V_{0}=-36.3$ and $A=1.1$ are given in Figure 4 The computation domain is chosen to be $[-32,32]^{2}$, due to the large support of the field profiles in the defocusing case. As before, we discretize the domain with 6 points per unit length and Figure 4 (a) is the band structure of the linear problem. Figure 4 (b) plots the $\lambda-P$ curve for $\lambda \in(-24.5,-23.4)$, which lies in between the first and second bands. This curve is obtained by following two continuation paths, one from $\lambda=-24$ down to $\lambda=-24.5$ and the second from $\lambda=-24$ to $\lambda=-23.4$. 
For both paths, the initial guess of $u(x)$ at $\lambda=-24$ is taken to be a localized Gaussian profile with energy $P=0.4$. We notice that the curve obtained is quite different from the one reported in [3]. Figure 4 (c) plots the number of Newton iterations and the averaged number of preconditioned GMRES iterations for each Newton step, as a function of $\lambda$. Figure $4(\mathrm{~d})$ and (e) are the field profiles at $\lambda=-24.4844$ and $\lambda=-23.4187$, respectively. In both plots, the field profile $u(x)$ is quite extended since the $\lambda$ value is close to the boundary of the bottom and top energy bands of the linear operator, respectively.

\section{CONCLUSiOn AND REMARKS}

In this work, we provide a robust and efficient method for soliton calculations based on Newton iteration and sparsifying preconditioner for the linearized problem. The Newton iteration based method is much more robust than Pitviashvili type approaches.

As for possible extensions, while in this paper we solve the nonlinear eigenvalue problem with prescribed eigenvalue $\lambda$ and undetermined normalization, the sparsifying preconditioner can be also applied to the problem with fixed normalization $\|u\|=m$ and undetermined $\lambda$. In the latter case, the Newton iteration becomes

$$
\left(\begin{array}{l}
u^{n+1} \\
\lambda^{n+1}
\end{array}\right)=\left(\begin{array}{l}
u^{n} \\
\lambda^{n}
\end{array}\right)-\left(\begin{array}{cc}
-\Delta+L_{u^{n}-\lambda^{n}} & -u^{n} \\
T_{u^{n}} & 0
\end{array}\right)^{-1}\left(\begin{array}{c}
-\Delta u^{n}+N\left(x, u^{n}\right)-\lambda^{n} u^{n} \\
\left(\|u\|_{2}^{2}-m^{2}\right) / 2
\end{array}\right) .
$$

Here $L_{u}$ is the linearization of the potential terms at $u: L_{u}=V+\frac{\delta N}{\delta u}$ and $T_{u}$ takes the inner product with $u: T_{u} v=\langle u, v\rangle$. In this case, the key step is the solution to the linear system

$$
\mathscr{L}_{(u, \lambda)}\left(\begin{array}{l}
\nu \\
\mu
\end{array}\right):=\left(\begin{array}{cc}
-\Delta+L_{u}-\lambda & -u \\
T_{u} & 0
\end{array}\right)\left(\begin{array}{l}
\nu \\
\mu
\end{array}\right)=\left(\begin{array}{l}
r \\
\kappa
\end{array}\right) .
$$

We can apply sparsifying preconditioner to solve this equation, and hence obtain an efficient algorithm.

Moreover, while in this work we focus on nonlinear Schrödinger equations arising from optics application, the method can be applied to other scenarios, for instance the nonlinear Maxwell equations. In addition, the sparsifying preconditioners also have potential applications in the context of Kohn-Sham density functional theory calculations. These directions will be explored in future works.

\section{REFERENCES}

[1] M. J. Ablowitz and Z. H. Musslimani, Spectral renormalization method for computing self-localized solutions to nonlinear systems, Opt. Lett. 30 (2005), 2140-2142.

[2] J. M. Dudley and J. R. Taylor, Ten years of nonlinear optics in photonic crystal fibre, Nature Photon. 3 (2009), 85-90.

[3] N. K. Efremidis, J. Hudock, D. N. Christodoulides, J. W. Fleischer, O. Cohen, and M. Segev, Two-dimensional optical lattice solitons, Phys. Rev. Lett. 91 (2003), 213906.

[4] A. George, Nested dissection of a regular finite element mesh, SIAM J. Numer. Anal. 10 (1973), 345-363. Collection of articles dedicated to the memory of George E. Forsythe. MR0388756 (52 \#9590)

[5] T. I. Lakoba and J. Yang, A generalized Petviashvili iteration method for scalar and vector Hamiltonian equations with arbitrary form of nonlinearity, J. Comput. Phys. 226 (2007), 1668-1692. 
[6] R. M. Martin, Electronic structure: Basic theory and practical methods, Cambridge, 2004.

[7] J. Nocedal and S. J. Wright, Numerical optimization, second ed., Springer, 2006.

[8] D. E. Pelinovsky and Y. A. Stepanyants, Convergence of Petviashvili's iteration method for numerical approximation of stationary solutions of nonlinear wave equations, SIAM J. Numer. Anal. 42 (2004), 1110-1127.

[9] V. I. Petviashvili, Formation of an extraordinary soliton, Sov. J. PLasma Phys. 2 (1976), 257.

[10] M. Plihal, A. Shambrook, A. A. Maradudin, and P. Sheng, Two-dimensional photonic band structures, Opt. Commun. 80 (1994), 199.

[11] Y. Saad and M. H. Schultz, GMRES: a generalized minimal residual algorithm for solving nonsymmetric linear systems, SIAM J. Sci. Statist. Comput. 7 (1986), 856-869.

[12] R. E. Slusher and B. J. Eggleton, Nonlinear photonic crystals, Springer Series in Photonics, Springer, 2004.

[13] M. Soljacic and J. D. Joannopoulous, Enhancement of nonlinear effects using photonic cyrstals, Nat. Mater. 3 (2004), 211-219.

[14] P. Tran, Photonic-band-structure calculation of material possessing Kerr nonlinearity, Phys. Rev. B 52 (1995), 10673.

[15] M. I. Weinstein, Localized states and dynamics in the nonlinear Schrödinger / Gross-Pitaevskii equation, Frontiers in Applied Dynamics: Reviews and Tutorials, Vol. 3, 2015, pp. 41-79.

[16] Lexing Ying, Sparsifying preconditioner for pseudospectral approximations of indefinite systems on periodic structures, Multiscale Model. Simul. 13 (2015), no. 2, 459-471. MR3332914

[17] __ Sparsifying preconditioner for the Lippmann-Schwinger equation, Multiscale Model. Simul. 13 (2015), no. 2, 644-660. MR3359667

Department of Mathematics, Department of Physics, Department of Chemistry, and Fitzpatrick Institute of Photonics, Duke University, Box 90320, Durham NC 27708, USA

E-mail address: jianfeng@math.duke.edu

Department of Mathematics and Institute of Computational and Mathematical Engineering, Stanford University, 450 SerRa MALL, BLDg 380, STANFord CA 94305, USA

E-mail address: lexing@stanford.edu 